\title{
Encoding processes and the recall of text
}

\author{
NEFF WALKER and J. PATRICK JONES \\ Teachers College, Columbia University, New York, New York 10027 \\ and \\ HARVEY H. MAR \\ Children's Hospital, Boston, Massachusetts 02115
}

\begin{abstract}
Two experiments investigated the proposition that the amount of cognitive effort expended to encode information will be directly related to recall of that information. While previous research has shown that amount of processing may affect recall, these studies have generally drawn on the notion of an elaborated memory representation to explain their results. In this study, the amount of processing required to correctly interpret anaphoric relations was varied while the elaboration of the memory trace was held constant. These experiments employed a self-paced reading paradigm in which subjects read a series of short paragraphs and later were cued to recall the final sentence of each paragraph. It was found that recall was significantly improved when more processing was required to correctly interpret the anaphoric relationship expressed in the final sentence. These findings suggest that encoding processes can affect recall performance without elaboration of the memory representation.
\end{abstract}

Although the original formulation of the levels-ofprocessing framework (Craik \& Lockhart, 1972) has been the subject of considerable criticism (see Baddeley, 1978; Nelson, 1977), revised versions of the model have been proposed that attribute differences in recall to various encoding activities. These versions can generally be divided into two groups. The elaboration model, proposed by Anderson and his colleagues (Anderson \& Reder, 1979), claims that deeper processing at encoding results in the creation of a set of multiple propositions that facilitate recall of the information. The distinctiveness theory, advanced by Jacoby (Jacoby \& Craik, 1979; Jacoby, Craik, \& Begg, 1979), predicts that deeper processing at encoding results in a more distinct memory representation that has a greater probability of being recalled at time of retrieval. In the present article, both of these models will be outlined and the results of two experiments will be reported that test the adequacy of the models to account for differences in the retention of prose.

The elaboration model, as proposed by Anderson and Reder (1979), suggests that different encoding processes result in more or less elaborated memory traces. Passages or sentences vary in the degree to which they encourage readers to draw inferences about consequences, causes, or outcomes. When the reader is able to draw an inference, the inferred information is stored along with the explicitly stated information. The storage

We are grateful to Jeff Franks, Miriam Goldberg, and John Limber for their helpful comments on earlier drafts of this manuscript. The second author is now at the Professional Examination Service, 475 Riverside Drive, New York, New York 10115 of inferred and explicit information results in an elaborated memory trace. The degree to which a passage or sentence is elaborated upon depends in part on task demands, readers' prior knowledge, and other nontextual factors. Bradshaw and Anderson (1982) suggest that elaborated traces are more easily recalled for two reasons. First, the presence of an elaborated trace results in more network redundancy in memory. When there is a great deal of redundancy in a memory trace, the possibility for alternative paths in memory to reach the information is enhanced. The second possibility is called inferential redundancy. The availability of additional information within the memory trace enhances the reader's ability to retrieve the elaborated material and therefore to infer or reconstruct the to-be-remembered information. Bradshaw and Anderson state that reconstruction of the material can occur even if the specific material is inaccessible at the time of retrieval.

In support of their theory of elaborative processes in memory, Bradshaw and Anderson (1982) compared the recall of specific information presented in three different contexts. The first context, called the singlesentence context, presented a piece of information about a famous person. The second context, called the unrelated context, presented that same sentence in addition to two other pieces of information about that person. However, these two pieces of information were not specifically related to the information given in the single target sentence. The third condition, the related condition, again presented the target sentence about the person, but it also provided two sentences that allowed the reader to infer the specific information presented in the target sentence. The additional sen- 
tences either provided the cause or stated the consequences of the information contained in the target sentence. Using cued recall tasks, Bradshaw and Anderson (1982) found recall performances that supported their hypothesis. The related condition produced the highest level of recall for the target sentence. The unrelated sentence condition produced the lowest level of recall performance, with the single-sentence condition being significantly different from both. While these results certainly offer strong support of Anderson and Reder's (1979) position concerning the facilitative effect of elaboration on recall performance, they do not preclude the possibility that processes other than elaboration could enhance recall. Elaboration theory, although based on differences in processing at time of encoding, assumes that recall differences are due to different structures present in memory.

A second group of theories that seeks to explain the effects of encoding processes on later recall support the distinctiveness model. This model, advocated primarily by Jacoby (Jacoby \& Craik, 1979; Jacoby et al., 1979), suggests that improved recall performance may be due to processing differences that occur at encoding but do not require different memory traces. Proponents of this model argue that differences in recall performance are due to varying levels of distinctiveness for a particular memory trace rather than the presence of additional information stored in memory. The analogy of perceptual distinctiveness is useful in clarifying this theoretical claim. If an item is stored in a background such that a great deal of contrast between the item and its background is evident, the item should be recognized or perceived more readily. Jacoby and his colleagues maintain that similar processes occur in memory. If a piece of information is stored in memory and is distinctive or different from the information stored with it, that information will be more accessible at the time of retrieval. If, however, the information is stored with related information and, therefore, is less distinct, the probability of that particular piece of information being recalled will be low.

One problem with this theory, similar to the major criticism of the depth-of-processing model (Craik \& Lockhart, 1972), is the difficulties involved in obtaining an independent measure of distinctiveness or depth. Several variables that have been proposed, such as time, have been found to be inadequate measures of strength, effort, or in this case, distinctiveness.

Some previous research has sought to identify cognitive effort as a measure of distinctiveness or depth of processing (Johnson-Laird \& Bethell-Fox, 1978; JohnsonLaird, Gibbs, \& deMowbray, 1978; Tyler, Hertel, McCallum, \& Ellis, 1979). In these experiments, JohnsonLaird and others have attempted to show that the number of processes engaged in while encoding information affects the accessibility of that information in long-term memory. Johnson-Laird and Bethell-Fox
(1978) reported that subjects' recall of a question depended, in part, upon the difficulty level of the question. The more difficulty subjects experienced in arriving at an answer, the better was the recall of that question. These researchers hypothesized that the recall advantage noted for the more difficult questions was due to the extra processing involved in arriving at either a "yes" or "no" response to these questions.

While the work of Johnson-Laird and his colleagues (Johnson-Laird \& Bethell-Fox, 1978; Johnson-Laird et al., 1978) does show that extra processing increases the probability of correct recall, their work does not prove that the increased recall is due to the extra processing per se. Their results easily could be explained by assuming that the extra processing resulted in a more elaborated memory trace. To determine which of these explanations is correct, a procedure would have to be used that varies the amount of processing while holding constant the elaborateness of the memory trace.

We are proposing that Johnson-Laird and BethellFox's (1978) formulation regarding the number of steps involved in processing information is a valid measure of how distinctive the memory trace of that information will be. In order to test this assumption, we will borrow from the work of Haviland and Clark (1974). Haviland and Clark, along with numerous other researchers (e.g., Garrod \& Sanford, 1977; Yekovich \& Walker, 1978; Yekovich, Walker, \& Blackman, 1979) have postulated the processes readers must go through to calculate anaphoric relations. For example, if we take the two sentences (1) Bill ordered the spaghetti, and (2) The spaghetti tasted delicious, it is easy for readers to establish that the spaghetti mentioned in the second sentence is the same spaghetti as that mentioned in the first sentence. Clark (1977) says that (1) this process is guided by syntactic cues, such as the use of the specific article "the," and (2) because it is a simple, direct match from spaghetti as new information in the first sentence to spaghetti as old information in the second sentence, it is easy for readers to link the information in the first sentence to that in the second sentence and store both sentences in memory. However, if we change the first sentence in the above example to (1a) Bill ordered the food, a more complicated procedure must be followed for readers to connect the item "spaghetti" with its referent "food." In this instance, Clark states that readers must build a bridging structure that allows them to infer that the "spaghetti" mentioned in the second sentence is the "food" referred to in the earlier sentence.

Haviland and Clark (1974) found that it takes longer for one to read the last sentence "The spaghetti tastes delicious" when it has been preceded by a sentence containing an indirect referent (e.g., "food") than when it has been preceded by a sentence containing a direct referent (e.g., "spaghetti"). This finding is well supported by other researchers (Clark \& Sengul, 1979; Yekovich \& Walker, 1978). 
When one reads the above example, whether the first sentence contains a direct or an indirect referent, the memory representation will be very similar in terms of propositions. That is, although there is a one-word discrepancy between the two examples (food or spaghetti), the propositional representation in memory will be stored in terms of the direct referent. Thus, the procedure of calculating referents appears to provide an ideal test for investigating the validity of the distinctiveness hypothesis as it pertains to encoding activities. Using sentence passages similar to those developed by Haviland and Clark (1974), we will be able to systematically manipulate the amount of processing readers must perform to comprehend a final sentence by varying whether the referent in the previous sentence is a direct referent or an indirect referent.

This procedure will enable us to control a critical factor that Bradshaw and Anderson (1982) did not control for in their experiments. In their research, no attempt was made to hold constant the memory representations across conditions. That, of course, must be the case if one is hypothesizing elaboration as the sole reason for increased recall performance. In this study, we will systematically vary the amount of processing involved in reading a short passage, while maintaining a rough equality in terms of the elaborateness of the memory representations for the two conditions. In this manner, we should be able to test the assumption that different amounts or types of encoding processes affect the accessibility of information at the time of retrieval.

\section{EXPERIMENT 1}

The purpose of this study is to determine the applicability of these earlier findings to a reader's memory for prose. We will use the general paradigm of Haviland and Clark (1974) to discover if the processing differences caused by variations of anaphoric relations in text are reflected in differences in recall. A series of prose passages will be presented to subjects in which the type of referent is systematically varied. Again, it has been shown that when an item mentioned in text has an indirect (nonidentical) referent, it takes longer to comprehend than when the item has been preceded by a direct (identical) referent. This increase in reading time is believed to be due to the reader's need to build a bridging inference connecting an item to its indirect referent, or to relabel the individual in memory, or both. Through the use of these procedures, we will vary the processes used during the encoding of the text. A recall task will then be used to ascertain whether differences in processing at the time of encoding will be reflected in different levels of retention.

\section{Method}

Subjects. The subjects were 24 graduate students of education currently enrolled at Teachers College, Columbia Univer: sity. Subjects were paid $\$ 5$ for participating in the study. One subject who failed to follow instructions was eliminated from the subject pool and replaced by another recruited from the same population.

Materials. Thirty three-sentence passages were created for use in the experiment. The first and third sentences of a particular set were identical for each condition. The first sentence of each passage served as an introduction. The third sentence of each passage made a statement about the target item mentioned in the second sentence.

The second sentence differed for each of three conditions. The second sentence in the direct referent condition contained the specific target item that would be repeated in the third sentence. In the indirect referent condition, a general item was introduced in the second sentence. The second sentence in the no-referent condition contained information germane to the general topic of the discourse without mentioning either the specific or general target item. The following are examples of passages representing the three conditions. Sentence 1: The restaurant was quite crowded today. Sentence 2 (direct condition): One customer ordered a plate of spaghetti. Sentence 2 (indirect condition): One customer ordered a plate of food for his dinner. Sentence 2 (no-referent condition): Many customers had to wait before being served. Sentence 3: The spaghetti was quite delicious.

Counterbalancing of the stimulus passages was achieved by devising three orders such that one-third of the passages appeared under each condition. Thus there were 10 passages from each condition in each list.

Design and Procedure. Subjects were tested individually. Subjects were randomly assigned to one of three test lists. The 30 stimulus passages plus two practice stories were presented to the subjects one sentence at a time on a video monitor. All stimulus sentences were presented in the middle of the screen in uppercase letters. Subjects were told that in order to view a sentence, they would have to press the space bar on the Radio Shack TRS-80 microcomputer keyboard. They were told that as soon as they had read and understood the sentence, they were to press the space bar to receive a new sentence. A sentence informing the subjects that they had read the last sentence of a story appeared at the end of each passage. The microprocessor randomized passage presentation order and recorded reading times in milliseconds for each stimulus sentence.

After subjects had read all 30 passages, a statement signaling the onset of the cued recall task appeared on the screen. Subjects were informed that they would be cued with the first sentence of each story. Subjects were instructed to write down on a piece of paper whatever they could remember about the final sentence of that story. The presentation order of the sentence cues matched the presentation order of the stimulus passages. Subjects were asked to work at their own pace during the cued recall task and to press the space bar on the keyboard to receive a new cue.

The cued recall data were scored for the presence of the target item and for gist recall of the third sentence. One point was awarded for each target item mentioned. Subjects were credited one point for gist recall if their response reflected the general meaning of the third sentence. Protocols that contained errors in tense, pronoun usage, or target item specificity were not marked incorrect as long as the general meaning of the sentence was maintained. The recall protocols were scored independently by two judges. The interrater reliabilities ranged from .87 to .95 for the three conditions. Discrepancies in scoring were settled by agreement of the two judges.

Reading times were adjusted in two ways before they were subjected to analysis. 1 irst, all extreme latencies longer than $7 \mathrm{sec}$ were replaced; second, all latencies over 2.5 standard deviations from the subject's mean of each of the three conditions were replaced. Approximately $1 \%$ of the latencies were replaced following the procedure recommended by Winer (1971, p. 487).

\section{Results and Discussion}

Table 1 presents means and standard deviations of 
Table 1

Mean Reading Time (in Milliseconds) and Proportion Correct Recall of the Last Sentences in Experiment 1

\begin{tabular}{|c|c|c|c|c|c|c|}
\hline \multirow[b]{3}{*}{ Measure } & \multicolumn{6}{|c|}{ Condition } \\
\hline & \multicolumn{2}{|c|}{ Direct } & \multicolumn{2}{|c|}{ Indirect } & \multicolumn{2}{|c|}{ No Referent } \\
\hline & Mean & SD & Mean & SD & Mean & SD \\
\hline Reading Time & 1562 & 540 & 1696 & 483 & 1779 & 554 \\
\hline Correct Recall & .280 & .190 & .382 & .232 & .274 & .217 \\
\hline
\end{tabular}

reading times and recall scores for all conditions. A oneway, within-subjects, repeated-measures analysis of variance revealed a significant effect for condition $[F(2,46)=7.93, p<.001]$. Tukey a comparisons indicated a significant reading time advantage for direct passages as compared to indirect or no-referent passages.

The cued recall data, which constituted the dependent variable of primary interest, were scored for the presence or absence of the target item and for the gist recall of the third sentence. Table 1 contains summary statistics for the cued recall task by condition. An analysis of variance of target item recall indicated a significant effect for condition $[F(2,46)=3.58$, $\mathrm{p}<.05]$. Tukey a comparisons revealed a significant recall advantage for the indirect condition contrasted with the direct referent and the no-referent conditions $(p<.05)$. An analysis of variance was performed on the number of target items mentioned for each passage in each condition. The analysis yielded a nonsignificant $F$.

The gist recall results of the first experiment seem to provide very strong support for the position that qualitative differences in the memory trace affect recall performance. If one goes back to the example of direct and indirect passage types, it seems clear that more elaborated traces do not exist for the indirect passages. If one assumes that the memory trace for the passages is in the form of interconnected propositions (e.g., Kintsch \& van Dijk, 1978), there seems to be no way in which the recall results could be explained in terms of elaboration theory, for the propositional structure of the direct and indirect passages would differ only in the labeling of an element in a single proposition. However, three possible explanations do exist that could fit both these results and the elaboration theory.

The first is that higher recall in the indirect condition was due to more time being spent in reading (rehearsing) the last sentence. This explanation, although appealing in its simplicity, was tested for by the inclusion of the no-referent condition. Passages in the no-referent condition had the longest reading times for the target sentence, yet the recall performance was lowest in this condition. This pattern of results seems to eliminate a simple reading time/recall performance relationship.

The second possibility is that when a reader is required to make a bridging inference to correctly interpret an anaphoric relationship, this inference is stored along with the representation of the paragraph. If this is the case, then there would be a more elaborated memory representation for the paragraphs in the indirect referent condition.

The final way in which these results could be made to fit with an elaborated trace theory would be to assume a different structure in the memory representation of the passages. Earlier, we noted that if the passages were represented by a propositional chain there would be no real differences in the two memory traces. If, however, the memory traces of the passages were based upon the storage of main items or individuals (such as the theory suggested by Anderson \& Hastie, 1974, and Garrod \& Sanford, 1977), the results of the first experiment could be explained in terms of an elaborated memory trace.

Both Anderson and Hastie (1974) and Garrod and Sanford (1977) suggest that the information is stored in terms of the referential label that most specifically identifies the "individual." For example, if one reads the two sentences (1) The child was walking down the street, and (2) The little boy was obviously going home, after reading the first sentence, the information would be stored in terms of the "individual" labeled "child." Upon reading the second sentence, however, the new and old information would be stored in terms of the same individual but with a new, more specific label, "boy."

According to this system, in order for additional information to be stored in an individual location, it would have to be checked against the label "boy." Likewise, if one read (1) The boy was walking down the street, and (2) The boy was obviously going home, the memory representation would be centered around the individual labeled "boy." The difference in processing between these two sets of sentences would be the type of processing that occurred in arriving at the same memory representation. In the first instance, the reader will relabel the memory location, changing it from the general label "child" to the specific label "boy." In the second instance, no relabeling will occur; the specific label "boy" is used in both sentences. According to this theory of the memory representation of prose, the indirect passages used in the first experiment would have a slightly more elaborated memory trace.

While the use of elaboration theory to explain these results is possible, it is problematic. This explanation requires that a relatively small amount of "elaboration" must lead to a rather large increase (in this experiment, to more than a $25 \%$ increase) in recall performance. To check the validity of the first explanation above, a new factor was introduced in the second experiment. This factor was intended to eliminate any difference in memory representations of the text, while systematically varying the amount of processing required.

"Distance" will be the new factor used in the next experiment. The distance between a word that is 
assumed to be given and its referent has been found to affect processing during reading. Work by both Clark and Sengul (1979) and Garrod and Sanford (1977) has shown that it takes longer to comprehend a sentence when it refers to a referent mentioned two sentences previously than when it refers to a referent mentioned only one sentence previously. Clark and Sengul (1979) theorized that this was due to the fact that items one sentence back are in a privileged position in memory.

Clark and Sengul (1979) have explained this increase in reading time when the item is more than one sentence back in a passage by drawing upon Jarvella's (1971) discontinuity model. This discontinuity model is based on a theory of how much information a reader can actively deal with at any one time. In this model, the reader is able to actively handle the information presented in any given sentence, plus the information presented in the immediately previous sentence or clause. Therefore, any information presented in the immediately previous sentence is easily integrated with the information in the present sentence, either by direct matching or by building a bridging inference. If, however, information is not in the preceding sentence or clause but is several sentences back, it is no longer as easily connected by the reader to the current sentence. Thus, when one must find a referent that is no longer in a privileged memory location, an extra amount of processing must occur. This processing involves searching for and retrieving the referent from the memory representation of the paragraph. Clark and Sengul (1979) use this model to explain why it takes longer to comprehend a sentence when it refers to information that has been presented more than one sentence earlier. By varying the distance between a noun and its referent, we should be able to vary the amount of processing while eliminating any difference in the elaborateness of the memory representation. When a direct referent is no longer in a privileged memory location, an extra processing step will occur. However, no bridging inference will need to be made, nor will there be any differences in the wording of the paragraphs. The only difference that could exist between the memory representations of the paragraphs would be in terms of the ordering of propositions. The elaboration theory would clearly predict no recall differences due to distance between a noun and its referent. By introducing this new variable, distance, in the second experiment, a further test was made for the effects of qualitatively different encoding processes on recall performance.

\section{EXPERIMENT 2}

Experiment 2 was basically a replication of the first experiment. The new variable, distance, was introduced to determine if the recall results of the first experiment could be replicated and to determine if encoding differences that did not result in different memory traces would also be reflected by differences in recall performance.
Although this experiment orthogonally varied referent type (direct, indirect) and referent distance (near, far), the comparison of primary importance is between the recall levels of the sentences in the direct-near condition and the direct-far condition. It is within these two conditions that the amount of elaboration has been controlled for and the necessary encoding processes have been varied. It is hypothesized that recall of the target sentence will be higher in the direct-far than in the direct-near condition.

\section{Method}

Subjects. The subjects were 40 graduate students of education enrolled at Teachers College, Columbia University. Subjects were either paid $\$ 5$ for participating in the study or did so in order to fulfill a course requirement.

Materials. Forty four-sentence passages were created for use in the experiment. As in the first experiment, the first sentence always introduced a general topic and the last sentence used the target item as the subject of the sentence. The second and third sentences varied to create the four conditions. As in the first experiment, referent condition varied depending upon whether a general label or a specific label was used to precede the target item. Distance was varied by either placing the referent in the third sentence (near condition) or in the second sentence (far condition). The following are examples of passages representing the four conditions. Sentence 1 (all conditions): Bill was on his way to the store. Sentence 2 (near conditions): The streets were crowded with shoppers. Sentence 2 (direct-far): A truck ran through the red light. Sentence 2 (indirect-far): A vehicle ran through the red light. Sentence 3 (direct-near): A truck ran through the red light. Sentence 3 (indirect-near): A vehicle ran through the red light. Sentence 3 (far conditions): The streets were crowded with shoppers. Sentence 4 (all conditions): The truck hit a man in the crosswalk.

A direct-near passage had a sentence that was context related but did not mention the critical item in the second sentence slot. The third sentence in this condition introduced the specific item that would be repeated in the fourth sentence. Direct-far passages contained the same four sentences as the direct-near condition. However, the order of the second and third sentences was reversed. Increasing the distance between the target sentence and its referent was achieved by placing a filler sentence between the appearance of the specific target item in the second sentence and the reiteration of the target item in the fourth sentence.

An indirect-near passage resembled its direct counterpart with the exccption that the third sentence introduced a general item instead of a specific item. An indirect-far passage was created by reversing the order of the second and third sentences of the indirect-near version. Passages were counterbalanced across conditions, and each subject read 10 passages from each of the four conditions.

Design and Procedures. Subjects were tested individually. Each subject was randomly assigned to one of four stimulus lists. The procedures employed in this experiment were identical to those used in the first experiment. The experiment utilized a 2 by 2 within-subjects design, with referent type (direct-indirect) and distance (near-far) as the two factors.

Scoring of the cued recall task followed the same criteria employed in the first experiment. The interrater reliabilities between the two judges' scoring of the gist recall data ranged from .89 to .97 for the four conditions.

\section{Results and Discussion}

Means and standard deviations of reading times and recall performance are presented in Table 2. A two-way analysis of variance of the mean latencies revealed a significant main effect for referent type only $[F(1,39)=$ 
Table 2

Mean Reading Time (in Milliseconds), Proportion Correct Recall of the Last Sentences, and the Correlation Between Time and Recall in Experiment 2

\begin{tabular}{|c|c|c|c|c|c|c|c|c|}
\hline \multirow[b]{3}{*}{ Measure } & \multicolumn{8}{|c|}{ Condition } \\
\hline & \multicolumn{2}{|c|}{ Direct-Near } & \multicolumn{2}{|c|}{ Direct-Far } & \multicolumn{2}{|c|}{ Indirect-Near } & \multicolumn{2}{|c|}{ Indirect-Far } \\
\hline & Mean & SD & Mean & SD & Mean & SD & Mean & SD \\
\hline Reading Time & 1552 & 519 & 1588 & 540 & 1690 & 552 & 1721 & 578 \\
\hline Correct Recall & .285 & .210 & .345 & .250 & .345 & .228 & .303 & .230 \\
\hline Correlation & .098 & $.274^{*}$ & .304 & .028 & .253 & .057 & .320 & .022 \\
\hline
\end{tabular}

*Significance level.

$27.08, p<.001]$. The interaction between factors was not significant $[F(1,39)=.05]$.

A two-way analysis of variance revealed no significant main effects, but a significant interaction between referent type and referent distance was observed $[F(1,46)=$ $6.75, \mathrm{p}<.05]$. Follow-up analyses confirmed our hypothesis by revealing that both the direct-far and the indirect-near conditions led to significantly $(p<.05)$ higher levels of recall than did the direct-near condition. The number of target items mentioned for each condition was also subjected to an analysis of variance, which revealed no significant main effects for referent type $[F(1,39)=.14]$, distance $[F(1,39)=.06]$, or their interaction $[\mathrm{F}(1,39)=.20]$.

Table 2 also presents product-moment correlations between reading times and cued recall performance on the target sentences by condition. Recall of the fourth sentences was positively related to reading times in both far conditions and marginally related in the indirect-near condition.

As in Experiment 1, Experiment 2 demonstrated that subjects took longer to read a sentence in the indirect referent condition than in the direct referent condition. What was unexpected was the failure to find a main effect for distance on reading time. Given the findings of Clark and Sengul (1979), it is somewhat surprising that the distance between an item and its referent seemed to have little effect on processing time. However, closer examination of the experiments of Clark and Sengul revealed a high variability across experiments in terms of the amount of increased reading time due to referent distance. Also, there were several procedural differences (sentence length, presentation style) that may have accounted for the failure to find a significant main effect for distance. Given these drawbacks, it should be noted that there was a fairly consistent time difference for distance in both the direct and indirect referent conditions ( 36 and $31 \mathrm{msec}$ ).

The recall findings for the indirect-far condition were also unexpected in light of a cognitive effort approach to the explanation of qualitative encoding differences. It had been expected that the direct-far and indirect-near sentences would be recalled more successfully than direct-near sentences, and this expectation was upheld by the results. However, it was also expected that the indirect-far sentences would be recalled at least as successfully as the direct-far and indirect-near sentences, because they would be expected to demand the most processing on the part of the reader. The findings, however, show that the recall performance for indirectfar sentences fell between that of the direct-near sentences and the direct-far and indirect-near sentences.

How can these findings be explained? One possible explanation rests on the notion of a "blow-out" effect. This notion suggests that in the indirect-far condition the reader had to search for information in long-term memory and draw a bridging inference in order to correctly integrate the information. The processing demands were very large. In addition, it should be noted that the indirect-far condition violates standard rules concerning the relative locations in the text of a given item and its referent; for example, reading "The truck" at the start of the target sentence (note the earlier example) forces the reader to presuppose that there is a referent, but that referent was not available as expected in the preceding sentence. Thus the reader must either expend a great deal of cognitive effort to calculate the correct referent, or, as Haviland and Clark (1974) theorized, the reader can simply treat "The truck" as new information.

The intermediate level of recall performance under the indirect-far condition could thus be explained by the fact that readers were sometimes able to handle this double processing load and correctly integrate the information, while in other instances they were not able to do so. In the case in which the reader is able to locate the correct referent, a large amount of processing effort has been expended and, consequently, that reader performs well on the recall task. If, however, the reader spends processing time searching for a referent unsuccessfully and treats the item as new information, two differences in the results may be found. First, an unsuccessful search for a referent may require less processing time than a successful search, because the reader will not need to make the bridging inference to the item and will not relabel that item. Second, recall performance on items involved in these unsuccessful search procedures will be lower because the reader has not successfully 
integrated the material across sentences. This explanation could account for unexpected findings in the indirect-far condition. In some instances, readers were able to identify the referent (requiring greater amounts of processing time and leading to higher recall), but in other instances, the reader was not able to identify the referent (requiring less processing time and leading to lower recall), and the combination of these two different strategies led to the intermediate level of the results for the indirect-far condition as a whole. This explanation of the recall results of the indirect-far condition receives some support from the correlational data, for it was this condition that had the strongest positive correlation between reading time and correct gist recall.

\section{GENERAL DISCUSSION}

The experiments reported here provide strong evidence that qualitative differences in encoding processes lead to different levels of recall of the information. In both experiments, information that required more processing to be correctly encoded was recalled more frequently than that same information when it had been correctly encoded with less processing. This was true even when differences in the elaborateness of the memory trace were eliminated. The proposition that different amounts or types of encoding processes affect the retention and accessibility of structurally identical memory traces as suggested by the original levels-ofprocessing model (Craik \& Lockhart, 1972) and the distinctiveness model (Jacoby \& Craik, 1979) seems valid.

This is not to suggest that either of these models specifically supports or predicts the results obtained in this study. Rather, it is a validation of the overall orientation of the two models. With regard to the results of this study, the distinctiveness model of Jacoby and Craik (1979) specifically states that amount of processing effort or number of processing steps is not a measure of distinctiveness. However, both the distinctiveness model and its earlier counterpart, the levels-of-processing model, fail to identify any independent measure of distinctiveness.

One possible shortcoming of the results of this study lies in the interpretation of Anderson's (e.g., Anderson \& Reder, 1979; Bradshaw \& Anderson, 1982) associative model of memory. According to our interpretation, the memory representations of a paragraph in the direct-near or the direct-far condition would be identical. However, another interpretation of the model might represent the two memory traces in such a way that the traces would differ in amount of network redundancy. As noted in the introduction. Anderson and Reder (1979) state that increasing network redundancy will also increase the probability of recall. In our view, this caveat does not vitiate the findings of the research reported here. It does, however, highlight the need for further research that investigates the possibility that network redundancy will enhance recall performance.

The results of these experiments support recent work on cognitive effort (e.g., Tyler et al., 1979; JohnsonLaird \& Bethell-Fox, 1978). The work of these researchers suggests that the amount of cognitive effort or processing required to encode information determines how readily that information will be recalled. However, most of the research in this area has failed to control for structural differences in the resulting memory trace (such as elaboration) and have therefore failed to show clear qualitative encoding effects. In addition, the experiments in the current study, unlike most of the earlier work in cognitive effort, have used methods that are typical of the processes that occur in normal reading. By showing that the difficulty involved in inferring or finding the correct anaphoric relationship affects recall performance, this study has shown that qualitative encoding differences do affect the retention of textual information.

However, these findings do not vitiate all of the claims made by the elaboration theory. The claim that elaborated representations lead to better recall seems valid. If, on the other hand, the claim is that elaboration explains all encoding effects on later recall, the elaboration theory is overstated. We are suggesting that different types or amounts of processing during encoding affect later recall in two ways. First, the encoding processes result in a more elaborated or quantitatively larger memory representation. This could lead to better recall due to either increased network or inferential redundancy. Second, the additional amount or type of processing results in qualitatively different memory representations. This difference can be in the form of trace strength, which is directly proportional to the amount of encoding processing performed. Trace strength, along with other variables such as elaborateness of the memory representation and the retrieval environment, would interact to determine the accessibility of information for later recall.

\section{REFERENCES}

Anderson, J., \& Hastie, R. Individuation and reference in memory: Proper names and indefinite descriptions. Cognitive Psychology, 1974, 6, 495-514.

Anderson, J. R., \& ReDER, L. M. An elaborative processing explanation of depth of processing. In L. Cermak \& F. Craik (Eds.), Levels of processing in human memory. Hillsdale, N.J: Erlbaum, 1979.

Baddeley, A. D. The trouble with levels: A re-examination of Craik and Lockhart's framework for memory research. Psychological Review, 1978, 85, 139-152.

Bradshaw, G. L., \& Anderson, J. R. Elaborative encoding as an explanation of levels of processing. Journal of Verbal Learning and Verbal Behavior, 1982, 21, 165-174.

Clark, H. H. Inferences in comprehension. In D. LaBerge \& S. J. Samuels (Eds.), Basic processes in reading: Perception and comprehension. Hillsdale, N.J: Erlbaum, 1977.

Clark, H. H., \& Senaul, C. J. In search of referents for nouns and pronouns. Memory \& Cognition, 1979, 7, 35-41. 
Craik, F. I. M., \& Lockhart, R. S. Levels of processing: A framework for memory research. Journal of Verbal Learning and Verbal Behavior, 1972, 11, 671-684.

GARROD, S., \& SANFORD, A. Interpreting anaphoric relations: The integration of semantic information while reading. Journal of Verbal Learning and Verbal Behavior, 1977, 16, 77-90.

Haviland, S. E., \& ClaRk, H. H. What's new? Acquiring new information as a process in comprehension. Journal of Verbal Learning and Verbal Behavior, 1974, 13, 512-521.

JACOBY, L. L., \& CraIK, F. I. M. Effects of elaboration of processing at encoding and retrieval: Trace distinctiveness and recovery of initial content. In L. Cermak \& F. Craik (Eds.), Levels of processing in human memory. Hillsdale, N.J: Erlbaum, 1979.

Jacoby, L. L., Craik, F. I. M., \& Begg, I. Effects of decision difficulty on recognition and recall. Journal of Verbal Learning and Verbal Behavior, 1979, 18, 585-600.

JARVELLA, R. J. Syntactic processing of connected speech. Journal of Verbal Learning and Verbal Behavior, 1971, 10, 409-416.

Johnson-Laind, P. N., \& Bethell-Fox, C. E. Memory for questions and amount of processing. Memory \& Cognition, $1978,6,496-501$.
Johnson-Laird, P. N., Gibes, G., \& deMowbray, J. Meaning, amount of processing, and memory for words. Memory \& Cognition, 1978, 6, 372-375.

KINTSCH, W., \& vSN DiJK, T. W. Towards a model of text comprehension and production. Psychological Review, 1978, 85, 363-394.

Nelson, T. O. Repetitions and depth of processing. Journal of Verbal Learning and Verbal Behavior, 1977, 16, 151-171.

Tyler, S. W., Hertel, P. T., McCallum, M. C., \& Ellis, E. C. Cognitive effort and memory. Journal of Experimental Psychology: Human Learning and Memory, 1979, 6, 607-617.

Winen, B. J. Statistical principles in experimental designs. New York: McGraw-Hill, 1971.

Yekovich, F. R., \& WALKen, C. H. Identifying and using referents in sentence comprehension. Journal of Verbal Learning and Verbal Behavior, 1978, 17, 265-277.

Yekovich, F. R., Walker, C. H., \& Blackman, H. S. The role of presupposed and local information in integrating sentences. Journal of Verbal Learning and Verbal Behavior, 1979, 18, 535-548.

(Received for publication August 2, 1982; revision accepted January $3,1983$. 